\title{
ON THE PERIODIC POINTS OF A TYPICAL CONTINUOUS FUNCTION
}

\author{
K. SIMON
}

(Communicated by R. Daniel Mauldin)

\begin{abstract}
Let $n$ and $k$ be arbitrary natural numbers. We prove that for a typical continuous function $f$, every neighborhood of any periodic point of $f$ with period $n$ contains periodic points of $f$ with period $n \cdot k$.
\end{abstract}

The purpose of this paper is to examine the structure of the set of periodic points of typical continuous functions. We shall denote by $C$ the set of continuous functions mapping the interval $[0,1]$ into itself. This set becomes a metric space with the supremum metric. By the term "typical continuous function" we mean that the set of all functions having the property under consideration is a residual subset of the metric space $C$.

In [1] S. J. Agronsky, A. M. Bruckner, and M. Laczkovich proved that for a typical continuous function $f$ any neighborhood of a periodic point contains periodic points of arbitrarily large periods. In this paper we prove that this result is true if "arbitrarily large periods" is replaced by "period $k \cdot n(k=1,2, \ldots)$ " (Theorem 1). As a consequence, for a typical continuous $f$ we have that $P_{f}^{n}$, the set of periodic points with period $n$, is uncountable, dense in itself, but not closed. Also, $P_{f}^{n}$ is a residual subset of $\operatorname{Fix}\left(f^{n}\right)$.

In [1] it is proved that, for a typical continuous $f, \operatorname{Fix}\left(f^{n}\right)$ is nowhere dense and perfect. In this paper we prove (Theorem 2) that $\operatorname{Fix}\left(f^{n}\right)$ is bilaterally strongly $\Phi$-porous. For $f \in C$ and $n \in \mathbf{N}$ we define $f^{n}(x)$ by $f^{1}(x)=f(x)$ and $f^{n}(x)=f\left(f^{n-1}(x)\right) . \quad P_{f}^{n}$ denotes the set of periodic points of $f$ with period $n$. We denote $\operatorname{Fix}\left(f^{n}\right)=\left\{x: f^{n}(x)=x\right\}$; then $P_{f}^{n}=\operatorname{Fix}\left(f^{n}\right) \backslash \bigcup_{k<n} \operatorname{Fix}\left(f^{k}\right)$. In particular $P_{f}^{n}$ is a relatively open subset of Fix $\left(f^{n}\right)$. For $f \in C$ and $\varepsilon>0$ denote

$$
B(f, \varepsilon)=\{g \in C:\|f-g\|<\varepsilon\} .
$$

Theorem 1. Let $n, k \in \mathbf{N}$ be arbitrary. Then for a typical continuous function $f$, every neighborhood of any periodic point of $f$ with period $n$ contains periodic points of $f$ with period $n \cdot k$.

Received by the editors August 3, 1987.

1980 Mathematics Subject Classification (1985 Revision). Primary 54H20; Secondary 26A18. 
Proof. Fix $n \in \mathbf{N}$. Let $A_{0}=\left\{f \in C: \operatorname{Fix}\left(f^{n}\right)\right.$ nowhere dense $\}$ and $A_{i}=\{f \in$ $C$ : every neighborhood of any periodic point of $f$ with period $i$ contains, for every $m \in \mathbf{N}$, periodic points of $f$ with period $m \cdot i\}(i=1,2, \ldots)$.

We prove by induction that each $A_{i}$ is residual. In [1] it is proved that the set $A_{0}$ is a residual subset of $C$. Assume that $\bigcap_{0 \leq i<n} A_{i}$ is a residual subset of $C$. We shall prove that $A_{n}$ is also residual. If $f \in \bigcap_{0 \leq i<n} A_{i}$ and $I$ is an arbitrary open interval then $P_{f}^{n} \cap I=\varnothing$ implies $\operatorname{Fix}\left(f^{n}\right) \cap I=\varnothing$. For $k \in \mathbf{N}$ and $\varepsilon>0$ we define the set of $Q_{k}^{\varepsilon}$ by

$$
Q_{k}^{\varepsilon}=\left\{f \in C \text { : for any } x_{0} \in P_{f}^{n} \text { there is } x \in P_{f}^{k \cdot n} \text { such that }\left|x-x_{0}\right|<\varepsilon\right\} .
$$

It will be sufficient to show that each $Q_{k}^{\varepsilon}$ contains a set which is open and dense. Let $f \in \bigcap_{0 \leq i<n} A_{i}$ and let $\varepsilon^{\prime}>0$ be arbitrary. We prove that there exist $g \in B\left(f, \varepsilon^{\prime}\right)$ and $\eta>0$ such that $B(g, \eta) \subset Q_{k}^{\varepsilon}$. Let $\left\{a_{i}\right\}_{i=0}^{l} \subset[0,1] \backslash \operatorname{Fix}\left(f^{n}\right)$ be a finite sequence such that $a_{0}=0, a_{l}=1$ and $0<a_{i+1}-a_{i}<\varepsilon$ for every $0 \leq i<l$. Put $J_{i}=\left[a_{i-1}, a_{i}\right](i=1, \ldots, l)$. Choose a finite sequence $\left\{b_{j}\right\}_{j=1}^{z} \subset P_{f}^{n}$ as follows: if $J_{i} \cap P_{f}^{n} \neq \varnothing(i=1, \ldots, l)$ then there should exist an $x_{0} \in J_{i} \cap P_{f}^{n}$ such that the sequence $\left\{b_{j}\right\}_{j=1}^{z}$ contains the orbit of $x_{0}$. Let $z^{\prime}=z / n$. We can choose $b_{1}, b_{2}, \ldots, b_{z}$ so that they are contained in different orbits. Choose $\eta_{1} \in \mathbf{R}$ so that

(i) $\eta_{1}>0$, (ii). $\eta_{1}<\frac{1}{3} \cdot \min \underset{\substack{0 \leq i_{1}<i_{2} \leq l \\ 0 \leq j_{2}<j_{2} \leq z \\ 0 \leq i \leq l, 0 \leq j \leq z}}{ }\left\{\left|a_{i_{1}}-a_{i_{2}}\right|,\left|b_{j_{1}}-b_{j_{2}}\right|,\left|a_{i}-b_{j}\right|\right\}$,

(iii) $\eta_{1}<\varepsilon^{\prime} / 3$, and

(iv) if for some $i \quad J_{i} \cap P_{f}^{n}=\varnothing$ and $\|g-f\|<3 \eta_{1}$ then $J_{i} \cap P_{g}^{n}=\varnothing$.

This is possible since $f \in \bigcap_{i<n} A_{i}$. Let $0<u<\eta_{1}$ be such that if $|x-y|<u$ then $|f(x)-f(y)|<\eta_{1}$. Then $\left[b_{i}-u / 2, b_{i}+u / 2\right] \cap\left[b_{j}-u / 2, b_{j}+u / 2\right]=\varnothing$ for every $1 \leq i<j \leq z$.

We can now define the function $g \in C$. First we define $g$ on

$$
\bigcup_{i=1}^{n}\left[f^{i}\left(b_{m}\right)-u / 2, f^{i}\left(b_{m}\right)+u / 2\right] \quad\left(m=1, \ldots, z^{\prime}\right)
$$

using the following procedure. Let $1 \leq m \leq z^{\prime}$ be fixed but arbitrary and take $x_{0}=b_{m}$. Put $I_{j}=\left[f^{j-1}\left(x_{0}\right)-u / 2, f^{j-1}\left(x_{0}\right)+u / 2\right]$. Let us define $g$ on the set $\bigcup_{j=1}^{n} I_{j}$ so that the graph of $g$ on this set will be included in the squares $E_{j}=I_{j} \times I_{j+1}$ (see Figure 1). If $j>1$ then let the graph of $g$ in $E_{j}$ be the increasing diagonal of $E_{j}$. (See Figure 1.) Let $x_{1}, \ldots, x_{k}, x_{k+1}=x_{1}$ be distinct points in $(0, u)$ and let the point $W_{i}$ be defined by $W_{i}=\left(x_{i}, x_{i+1}\right)$ $(i=1, \ldots, k)$; then $W_{i} \in L=[0, u] \times[0, u]$. Let $v=\min _{i \neq j} \frac{1}{5}\left|x_{i}-x_{j}\right|$ and define

$$
B_{i}=\left[x_{i}-\frac{v}{2}, x_{i}+\frac{v}{2}\right] \times\left[x_{i+1}-\frac{v}{2}, x_{i+1}+\frac{v}{2}\right] .
$$


$n=4, k=3$

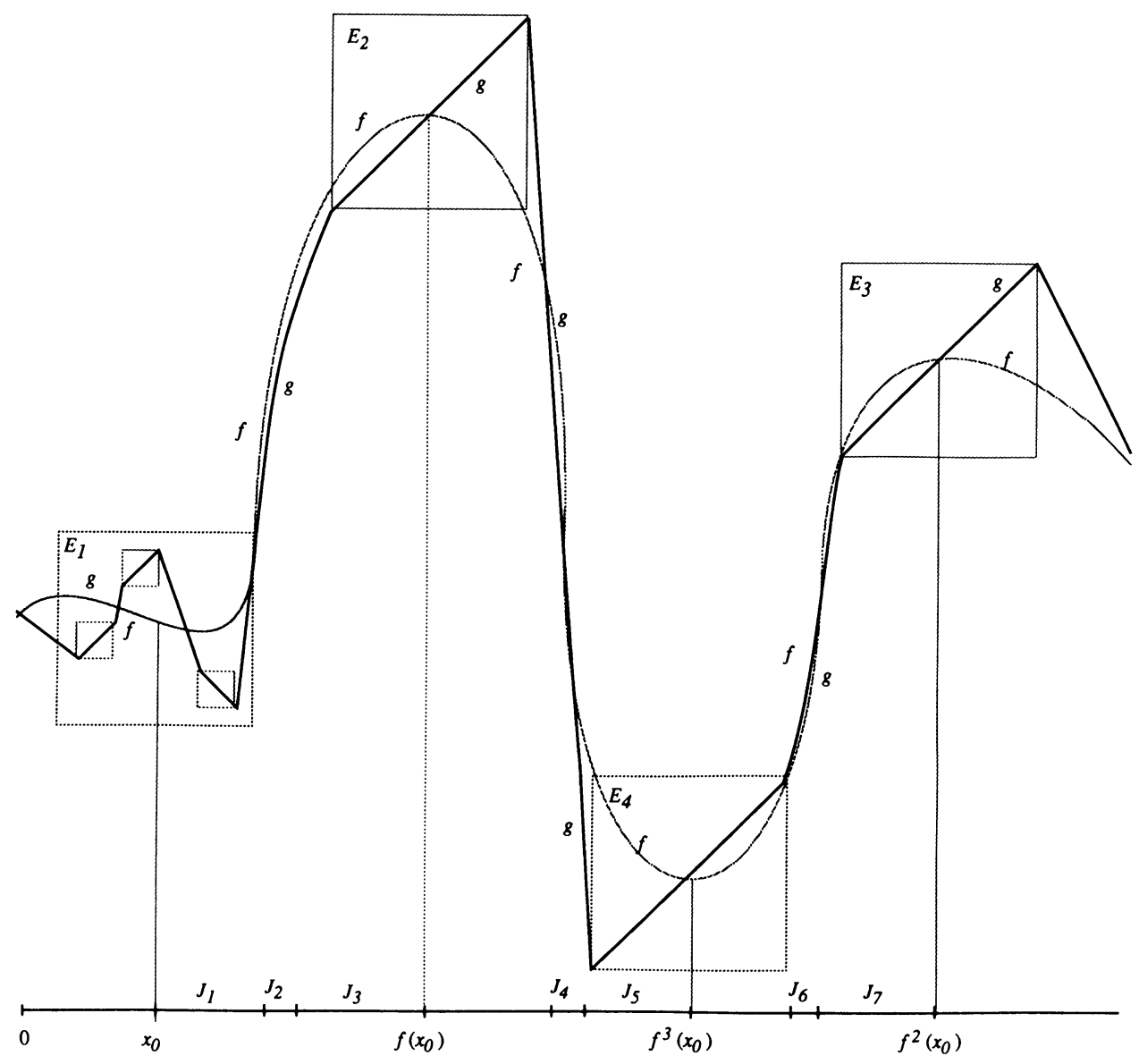

FIGURE 1

Let $h:[0, u] \rightarrow[0, u]$ be piecewise linear and continuous such that the graph of $h$ in each $B_{i}$ is the diagonal of $B_{i}$, if $1 \leq i<k$ then $h$ is increasing in $B_{i}$ but $h$ is decreasing in $B_{k}$. (See Figure 2.)

It is easy to see that if $\eta=v / 10$ and $q \in B(h, \eta)$ then $q$ has periodic points of period $k$ in the interval $\left(x_{i}-v / 2, x_{i}+v / 2\right)$. Now we define the graph of $g$ on $I_{1}$ by translating the graph of $h$ into $E_{1}$ (using the translation that maps $L$ onto $E_{1}$ ). Thus we have defined $g$ on $\bigcup_{i=1}^{2}\left[b_{i}-u / 2, b_{i}+u / 2\right]$. Put $g\left(a_{i}\right)=f\left(a_{i}\right) \quad(i=0,1, \ldots, l)$. If $J_{i} \cap P_{f}^{n}=\varnothing$ then let $\left.g\right|_{J_{i}}=\left.f\right|_{J_{i}}$. For those $x$ 's for which $g$ has not yet been defined, let us define $g$ so that $\|f-g\|<\eta_{1}$; by the definition of $u$ this is possible. Now if $P_{f}^{n} \cap J_{i}=\varnothing$ then $P_{g}^{n} \cap J_{i}=\varnothing$ or else $P_{g}^{n \cdot k} \cap J_{i} \neq \varnothing$. Thus $g \in B\left(f, \varepsilon^{\prime}\right) \cap Q_{k}^{\varepsilon}$ and it is easy to see that if $\eta=v / 10$ then $B(g, \eta) \subset B\left(f, \varepsilon^{\prime}\right) \cap Q_{k}^{\varepsilon}$. It follows that $Q_{k}^{\varepsilon}$ contains 


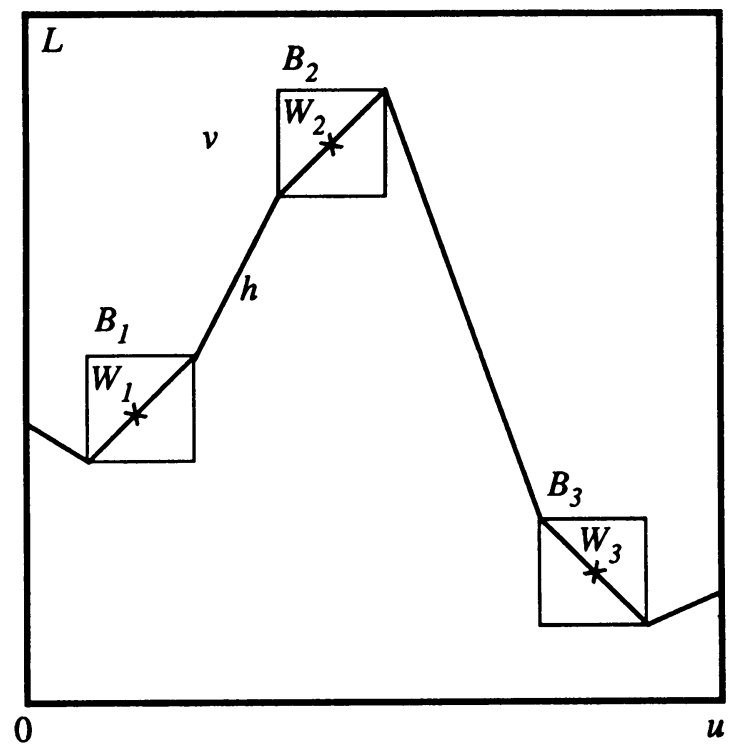

FiguRe 2

an open dense set, and since $\bigcap_{\varepsilon, k} Q_{k}^{\varepsilon} \subset A$ we have that $A$ is a residual subset of $C$. This completes the proof of the theorem.

We show that, for a typical continuous $f, P_{f}^{k}$ is a dense relatively open subset of $\operatorname{Fix}\left(f^{k}\right)$ from which it will follow that, $P_{f}^{k}$ is a residual subset of Fix $\left(f^{k}\right)$ and $P_{f}^{k}$ is uncountable.

Corollary 1. For a typical continuous $f, P_{f}^{k}$ is a dense relatively open subset of $\operatorname{Fix}\left(f^{k}\right)$.

Proof. If $x \in \operatorname{Fix}\left(f^{k}\right)$ then there is $\left.q\right|_{k}$ such that $x \in P_{f}^{q}$. It follows from Theorem 1 that any neighborhood of $x$ contains periodic points with period $q \cdot k / q$. As we have seen in the introduction, $P_{f}^{k}$ is relatively open in $\operatorname{Fix}\left(f^{k}\right)$.

Corollary 2. For a typical continuous $f, P_{f}^{k}$ is not closed for every $k>1$.

Proof. It follows from Theorem 1 that for every $x \in \operatorname{Fix}(f), x \in \operatorname{cl}\left(P_{f}^{k}\right)$ and $x \notin P_{f}^{k}$.

Corollary 3. For a typical continuous $f, P_{f}^{k}$ is dense in itself.

Proof. Since, for a typical $f, \operatorname{Fix}\left(f^{k}\right)$ is dense in itself [1], the statement follows from Corollary 1. 
Remark. For a typical $f$ there is $x \in \operatorname{Fix}\left(f^{n}\right)$ such that in a suitable neighborhood of $x$, the periods of the periodic points are all multiples of $n$. Moreover, this is true for the points of a residual subset of $\operatorname{Fix}\left(f^{n}\right)$. Indeed, let $f \in C$ and $I_{0} \subset[0,1]$ be an open interval and let $I_{k}=f^{k}\left(I_{0}\right)$. Define $P_{k}^{n}(f)=\left\{I_{0}:\right.$ for every $0 \leq 1 \neq j \leq k-1, I_{i} \cap I_{j}=\varnothing$ and $I_{n} \subset I_{0}$ and $\min \left\{\left|I_{0}\right|, \ldots,\left|I_{n}\right|\right\}<1 / k$. (See [1, Proposition 1].)

In [1] the authors proved that for a typical continuous function $f, \operatorname{Fix}\left(f^{n}\right) \cap$ $\bigcap_{k=1}^{\infty} G_{k}^{n}$ is a residual subset of $\operatorname{Fix}\left(f^{n}\right)$. Put $B_{f}^{n}=\left\{x \in P_{f}^{n}\right.$ : there is $V$ neighborhood of $x$ such that for any periodic point $y \in V$ there is $k$ such that period of $y$ equal to $n \cdot k\}$. It is easy to see $P_{f}^{n} \cap \bigcap_{k=1}^{\infty} G_{k}^{n} \subset B_{f}^{n}$ and therefore $B_{f}^{n}$ is a residual subset of $\operatorname{Fix}\left(f^{n}\right)$.

In [1] it is proved that for a typical continuous $f$ the set $\operatorname{Fix}\left(f^{k}\right)$ is nowhere dense and perfect. In Theorem 2 we shall prove that for every porosity premeasure (i.e., a continuous map $\Phi:(0,1] \rightarrow(0,1]), \operatorname{Fix}\left(f^{k}\right)$ is bilaterally strongly $\Phi$-porous for a typical continuous $f$. This means that for every $x \in \operatorname{Fix}\left(f^{k}\right)$ there are sequences of intervals $\left\{I_{n}\right\}_{n=1}^{\infty}$ and $\left\{J_{n}\right\}_{n=1}^{\infty}$ such that $I_{n} \subset(x-1 / n, x) \backslash \operatorname{Fix}\left(f^{k}\right), J_{n} \subset(x, x+1 / n) \backslash \operatorname{Fix}\left(f^{k}\right)$ and

$$
\lim _{n \rightarrow \infty} \frac{\operatorname{dist}\left(x, I_{n}\right)}{\Phi\left(\left|I_{n}\right|\right)}=\lim _{n \rightarrow \infty} \frac{\operatorname{dist}\left(x, J_{n}\right)}{\Phi\left(\left|J_{n}\right|\right)}=0 .
$$

We shall apply the method used by P. Humke and M. Laczkovich in [2] to this problem.

The pair of sequences $(\alpha, \beta)$ is said to be proper if $\alpha=\left\{\alpha_{n}\right\}_{n=1}^{\infty}, \beta=$ $\left\{\beta_{n}\right\}_{n=1}^{\infty}$ and $\beta_{n} \rightarrow 0$ and $0<\alpha_{n}<\beta_{n}$. If $(\alpha, \beta)$ is a proper pair of sequences then the sequence $x=\left\{x_{n}\right\}_{n=1}^{\infty}$ is called an $(\alpha, \beta)$-sequence if $x_{n} \rightarrow x_{0}$ and $\alpha_{n} \leq x_{n}-x_{0} \leq \beta_{n}$ for each $n \in \mathbf{N}$. Let the natural number $l>1$ and the proper pair $(\alpha, \beta)$ be fixed. (See [2, p. 245].) Put

$B_{N}=\left\{f \in C:\right.$ there is $\left\{x_{i}\right\}_{i=1}^{\infty}(\alpha, \beta)$-sequence such that $f^{l}\left(x_{i}\right)=x_{i}$ if $i \geq N\}$.

Lemma. $B_{N}$ is nowhere dense.

Proof. First we shall prove that $B_{N}$ is closed. Let $\left\{f_{k}\right\}_{k=1}^{\infty} \subset B_{N}$ and $f_{k} \rightarrow f$ in $C$. Thus, for every fixed $k$ there is a sequence $x^{k}=\left\{x_{n}^{k}\right\}_{n=1}^{\infty}$ converging to $x_{0}^{k}$ such that $x^{k}$ is an $(\alpha, \beta)$-sequence and $f_{k}^{l}\left(x_{i}^{k}\right)=x_{i}^{k}$ if $i \geq N$. Using a diagonal procedure we obtain a subsequence $\left\{k_{i}\right\}_{i=1}^{\infty}$ such that for every $n$ there is $x_{n}^{*}$ such that $\left\{x_{n}^{k_{i}}\right\}_{i=1}^{\infty} \rightarrow x_{n}^{*}$ and $x^{*}=\left\{x_{n}^{*}\right\}_{n=1}^{\infty}$ is a $(\alpha, \beta)$-sequence. As $\left\{f_{k}\right\}_{k=1}^{\infty} \rightarrow f$ in $C$ and since for $n>N \quad f_{k_{i}}^{l}\left(x_{n}^{k_{i}}\right)=x_{n}^{k_{i}}$ it follows that $f^{\prime}\left(x_{n}^{*}\right)=x_{n}^{*}$ if $n>N$.

If $p$ is a polynomial then $\left\{x: p^{l}(x)=x\right\}$ is finite and hence $p \notin B_{N}$. Since the set of polynomials is dense in $C$, the complement of $B_{N}$ is dense and, as $B_{N}$ is closed, $B_{N}$ is nowhere dense.

Let $\Phi:(0,1] \rightarrow(0,1]$ be continuous arbitrary fixed. 
Theorem 2. For a typical continuous $f, \operatorname{Fix}\left(f^{l}\right)$ is bilaterally strongly $\Phi$-porous set.

Proof. Let $(\alpha, \beta)$ be such that $\alpha_{n}=\beta_{n+1}, \beta_{n}<1 / n$ and $\Phi\left(\beta_{n}-\beta_{n+1}\right) \geq$ $n \beta_{n+1}$. Let $M=\left\{x: f^{\prime}(x)=x\right\}$. If $f \in \cup_{n} B_{n}$ then for each $x \in M$ there is a sequence $\left\{n_{i}\right\} \rightarrow \infty$ such that $M \cap\left[x+\alpha_{n_{i}}, x+\beta_{n_{i}}\right]=\varnothing$. Let $J_{i}=\left[x+\alpha_{n_{i}}, x+\beta_{n_{i}}\right]$. Thus $J_{i} \subset(x, x+1 / i) \backslash M$ and

$$
\operatorname{dist}\left(x, J_{i}\right)=\alpha_{b_{i}}=\beta_{n_{i}+1}<\frac{\Phi\left(\beta_{n_{i}}-\beta_{n_{i}+1}\right)}{n_{i}}=\frac{\Phi\left(\left|J_{i}\right|\right)}{n_{i}}
$$

and hence

$$
\lim _{n \rightarrow \infty} \frac{\operatorname{dist}\left(x, J_{i}\right)}{\Phi\left(\left|J_{i}\right|\right)}=0
$$

Using a similar procedure we can find a sequence $\left\{I_{i}\right\}_{i=1}^{\infty}$ such that

$$
I_{i} \subset(x-1 / i, x) \backslash M \text { and } \lim _{i \rightarrow \infty} \frac{\operatorname{dist}\left(x, I_{i}\right)}{\Phi\left(\left|I_{i}\right|\right)}=0 .
$$

This completes the proof of the theorem.

\section{ACKNOWLEDGMENT}

The author would like to thank Miklós Laczkovich for his useful advice.

\section{REFERENCES}

1. S. J. Agronsky, A. M. Bruckner and M. Laczkovich, Dynamics of typical continuous functions, J. London Math. Soc. (2) (to appear).

2. P. Humke and M. Laczkovich, Typical continuous functions are virtually nonmonotone, Proc. Amer. Math. Soc. 92 (1985), 244-248.

Department of Mathematics, Technical University, Miskolc, Hungary 